\title{
A Control Method of the Force Loading Electro-hydraulic Servo System Based on BRF
}

\author{
Jing-Wen FANG ${ }^{1, a,{ }^{*}}$, Ji-Shun $\mathrm{LI}^{1,2, \mathrm{~b}}$, Fang YANG ${ }^{1}$, Yu-Jun XUE ${ }^{2}$ \\ ${ }^{1}$ College of Mechatronics Engineering, Henan University of Science and Technology, Luoyang \\ 471003, China \\ ${ }^{2}$ Henan Key Laboratory for Machinery Design and Transmission System, Henan University of \\ Science and Technology, Luoyang 471003, China \\ a392071709@qq.com, b ljs_homework@163.com, \\ * Corresponding author
}

Keywords: Electro-hydraulic Servo System, Nonlinear, RBF Neural Network.

\begin{abstract}
The paper proposed a control method based on the electro-hydraulic servo loading system used on the bearing test rig for aircraft engine. Aiming at the nonlinear problem of the system, a control method is designed based on RBF neural network. This method uses the BRF algorithm of neural network on the online adjustment and correction of the PID parameters. Through the simulation, we compare the control effect of classic PID and RBF tuning. Results show that the BRF algorithm has high robustness, adaptive. Its track performance and adaptive control capacity are superior to the classic PID control.
\end{abstract}

\section{Introduction}

In recent years, our country has many breakthroughs in the high speed, heavy load bearing area. Our school as well as other colleges and universities, research institutions also developed a new generation of high speed rail bearing test rig. However, the bearings tested by these bearing test rigs are still single rotor structure; experimental rig is also a single drive system. But some of the major foreign high-speed bearing manufacturers such as Sweden SKF, Germany INA/FAG, Japan NTN, all kinds of test rigs are developed to collect the relevant data of the bearing, and according to the test data, to produce a high performance bearing. In order to make up for our country in this respect is not enough and to develop China's aviation industry rapidly. Therefore, the research and development of the bearing test rig has important strategic significance and practical value.

Electro-hydraulic servo control system has been widely used in materials, engineering, aerospace, construction, automobile, railway, and many other fields. Because of the high accuracy, fast response and high output power, stability and other characteristics. But the system dynamics are complex, because of the essentially nonlinear. It has uncertainty caused by time-varying parameter and external disturbances. ${ }^{[1-3]}$ So this control system must be designed to take effective measures to minimize or avoid adverse effects of nonlinear system. Adaptive anti-jamming ability of classic PID control is not suitable for nonlinear time-varying systems and cannot achieve satisfactory control effect.

In this control system, in order to simulate the actual working conditions of bearings, which need axial and radial loads, loads precision directly related to the performance of the measured bearing. To improve control precision, we introduced in the RBF neural network control. Neural network control has the high control precision, ability to control nonlinear systems, fast operation and can learn itself. So this paper is based on the RBF neural network to automatically adjust and improve the classic PID control so as to achieve the best effect. ${ }^{[4-7]}$

\section{Establishment of Mathematical Model}

The bearing test rig for aircraft engine is mainly used to simulate the working condition of bearing on aviation engine main shaft, includes the performance test that bearings withstand radial 
and axial static dynamic loading and the durability test. Hydraulic load control system block diagram is shown in Figure 1, the system is a closed-loop control system constituted by servo amplifiers, electro-hydraulic servo valves, hydraulic cylinders, force sensors and so on.

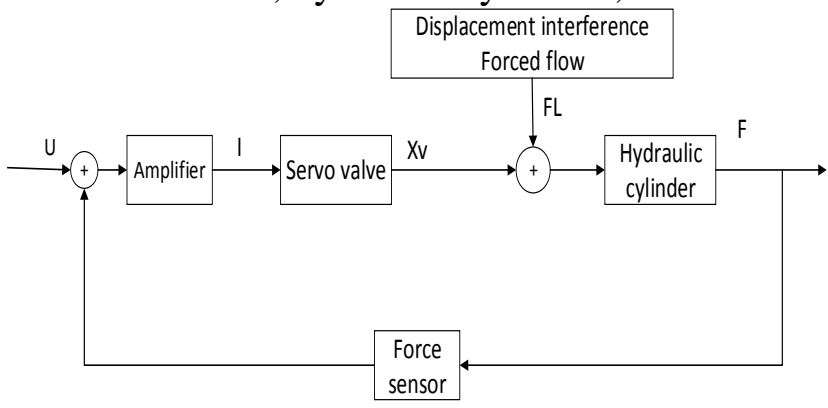

Fig.1 Block Diagram of Electro-hydraulic Servo System

In a servo system, mechanical clearances are common multivalued nonlinear characteristic. When it is in the control system, often leads to accumulation of energy, the surplus energy accumulation can cause system instability or limit cycle oscillations. In the course of development of electro-hydraulic servo control system of this test rig, we find that the forced flow caused by oil in cylinder squeezed by piston may induce the mechanical clearance between piston rod of hydraulic cylinder and load. This mechanical clearance can increase the steady-state error and the overshoot when system is under unit-step response. Because the test rig system we designed use the single thrust load and repeated fluctuating load and the existence of mechanical clearances, the system may cause reversal errors whenit changes the loading mode. These errors can affect the system tracking precision even make the control system invalidation.

Establish mathematical model of hydraulic servo system, preparing for analyzing double rotor bearing test rig for aircraft engine control system dynamic characteristics.

Flow equation of valve $Q_{L}=k_{q} x_{v}-k_{c} p_{l}$

Continuous flow equation $Q_{L}=A_{p} \frac{d x_{p}}{d t}+C_{t p} P_{l}+\frac{V_{t}}{4 \beta e} \frac{d P_{l}}{d t}$

Force balance equation $F=A_{P} P_{L}=m_{t} \frac{d^{2} x_{p}}{d t^{2}}+B_{P} \frac{d x_{p}}{d t}+K x_{p}+F_{L}$

Where $k_{q}$ means flow gain, $x_{v}$ means spool displacement, $k_{c}$ means flow-pressure coefficient, $A_{P}$ means hydraulic cylinder piston area, $m_{t}$ means mass of load, $x_{p}$ piston displacement, $\boldsymbol{B}_{p}$ means damping coefficient of the load, $C_{t p}$ means total leakage coefficient of hydraulic cylinder, $K$ means load spring, $P_{L}$ means supply pressure, $F_{L}$ means the disturbance on the piston rod, $V_{t}$ means the effective Piston of volume, $\beta e$ means the elastic modulus of the effective volume.

Simplify the control system block diagram without the outside interference; we can get the transfer function of the system.

$$
G(s) H(s)=\frac{K_{0} G_{s v}(s)}{\left(\frac{s}{\omega r}+1\right)\left(\frac{s^{2}}{\omega_{0}{ }^{2}}+\frac{2 \xi_{0}}{\omega_{0}} s+1\right)}
$$

Where $G_{S V}$ is the transfer function of the servo valve, generally use second-order turbulence links, $K_{0}$ is the open-loop gain of the system, $\omega_{r}$ is ratio of stiffness and damping coefficient between hydraulic spring and spring loaded which is serial coupled, $\omega_{0}$ is natural frequency formed from the stiffness between hydraulic spring and spring loaded which are serial coupled and mass of load, $\xi_{0}$ means damping ratio. Put the parameters in equation and we can get the transfer function of the system. 


\section{Controller Design}

In order to reduce the impact precision of nonlinear control system and to meet the requirements of control precision of the test rig. We decide to introduce the RBF neural network into classical PID control system. RBF based on neural network is a novel and efficient feed-forward neural network, its compensation control can do real time online learning on nonlinear uncertainty and also can do real-time compensation on parameter changes, disturbance and other uncertainty factors, these features can make up for classic compensation.

Neural network is a kind of mathematical model that can process signal by simulating biological neural systems. It composed by a large number of simple artificial neural through certain network structure and it is a kind of parallel work. The network structure is shown in Figure 2.

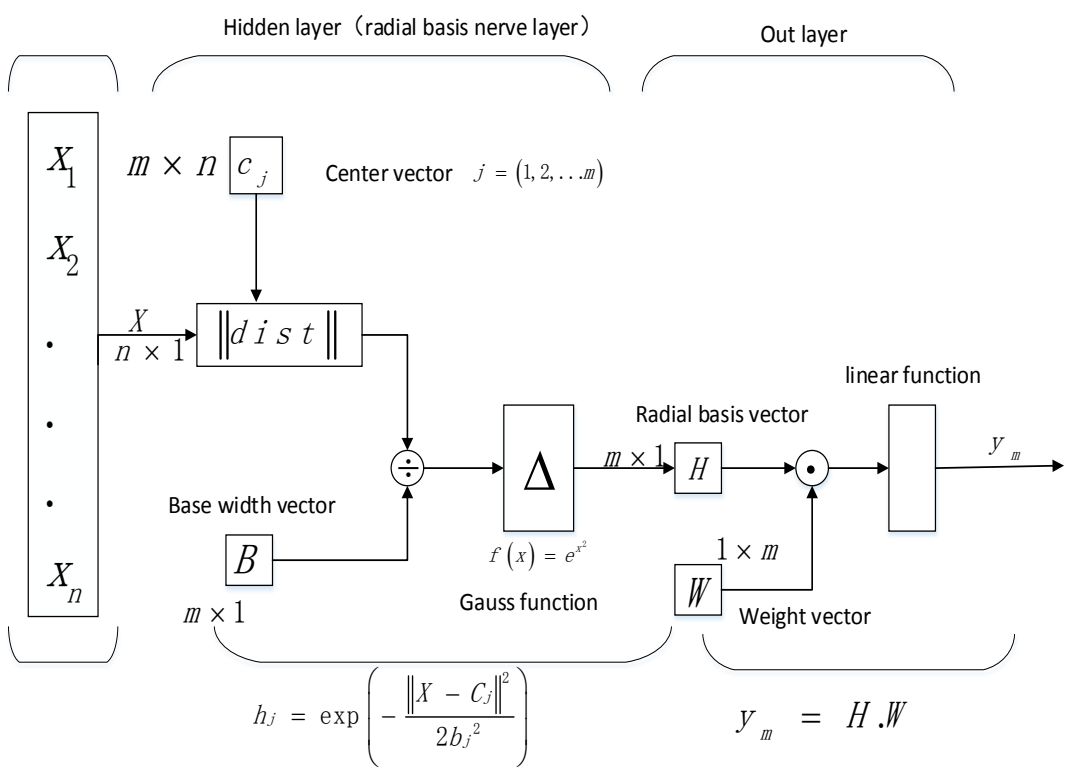

Fig.2 Structure of Neural Network

RBF neural network is a three-layer feed forward network with single-hidden layer, the first layer is input layer, composed by signal source node, the 2nd is hidden layer, the unit number by needs, the third layer to output layer, it responds to the input mode. Input layer nodes transmit signals to the hidden layer only, hidden layer nodes (RBF nodes), it composed by radiation effect function just like Gaussian function, but output layer nodes is usually a simple linear function.

In this paper, the $k_{p}, k_{i}, k_{d}$ is adjusted by gradient descent method. We use Lyapunov discrete function $V(t)=\frac{1}{2} E^{T} \cdot E$

$$
\begin{aligned}
& \text { Where } E(t)=\left[e_{1}(t), \ldots e_{N_{Y}}(t)\right] \\
& =\left[y_{1}{ }^{r}(t)-y_{1}(t), \ldots, \mathrm{y}_{N_{Y}}{ }^{r}(t)-y_{N_{Y}}(t)\right]^{T} \\
& =y^{r}(t)-y(t)
\end{aligned}
$$

Under the function of the $E(t)$ type definition, convergence theorem of neural network control algorithm with gradient descent algorithm.

Theorem is: if $\eta$ is Learning efficiency of neurons, $g_{\max }:=\max \|g(t)\|,\|\bullet\|$ means the Euclidean norm real space, $y$ means the network output. $\omega$ means the network power, so after verification, if $\mathrm{O}<\boldsymbol{\eta}<\frac{2}{\mathcal{S}^{2} \text { max }}$, can guarantee the convergence of the algorithm.

The RBF adjust block diagram of the PID control system is as follows in Figure 3. 


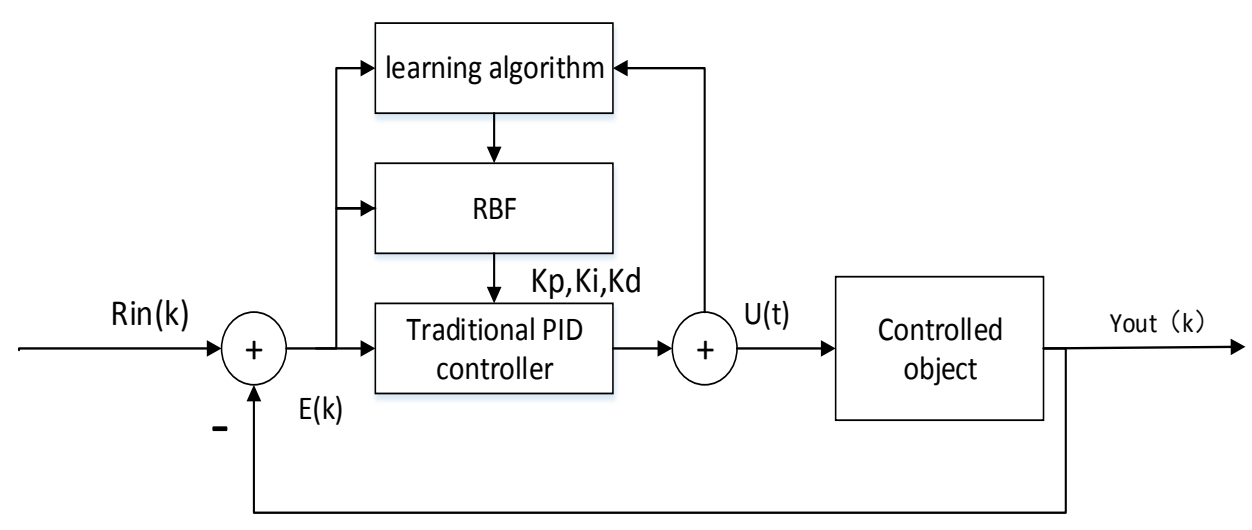

Fig.3 The RBF Adjust Block Diagram of the PID Control System

\section{Simulation and Analysis}

According to the control system of bearing test rig for aircraft engine model, we use the method of setting PID parameters to obtain the initial value of the neuron PID controller. We set learning efficiency $\eta$ as 0.25 , momentum factor $\alpha$ as 0.005 , proportional gain $k_{p}$ as 150 , integral control coefficient $k_{i}$ as 19 , differential control coefficient $k_{d}$ as 17. In MATLAB, simulating the PID parameter tuning control system is based on RBF neural network when the system is under the action of single thrust and alternating load. The simulation results are shown in Figure 4, 5, 6.
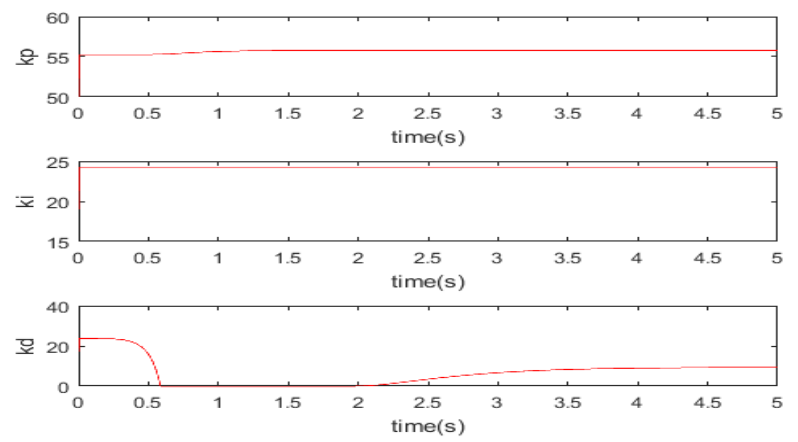

Fig.4 Parametric Adaptive Tuning Curve

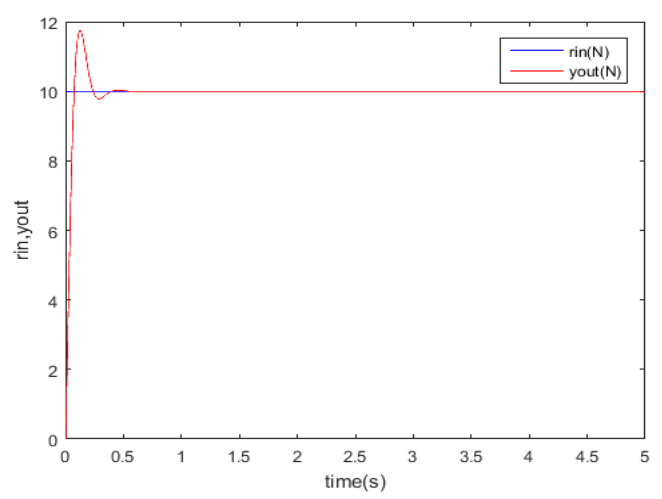

Fig.5 The Response Curve of RBF Tuning PID Control under Single Thrust

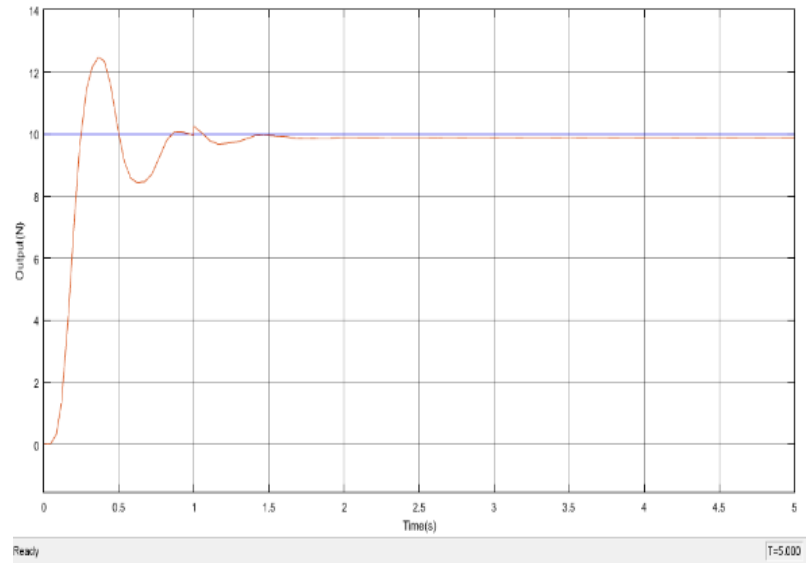

Fig.7 The Response Curve of Classic PID Control under Single Thrust 


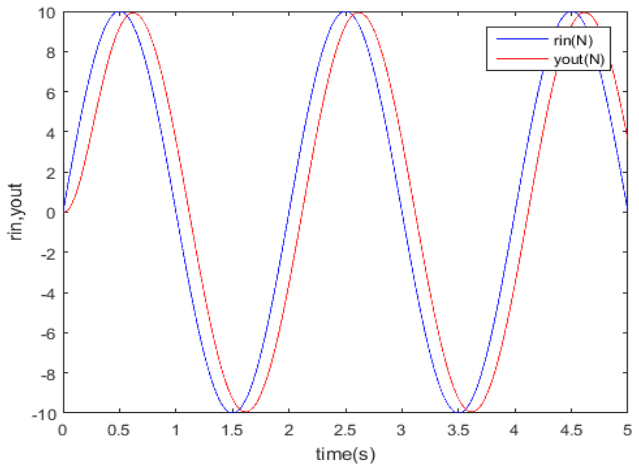

Fig.6 Control Response Curve of RBF PID under Alternating Load

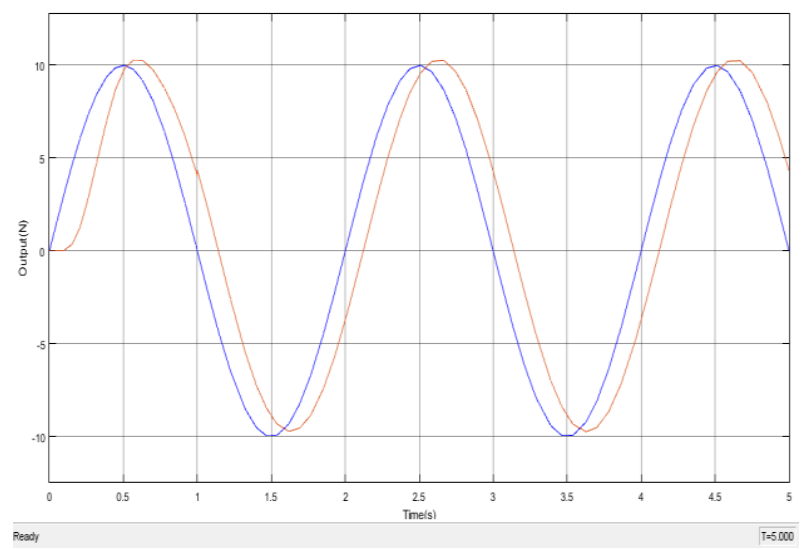

Fig.8 Control Response Curve of Classic Tuning PID under Alternating Load

The comparison between Figure 5, 6 and Figure 7, 8 (Output force image under the classic PID control of single thrust and alternating load) shows that, when under the PID control of RBF neural network tuning, the system's overshoot decreases, the rise time and adjusting time of the curve are greatly shortened, and the method restrain the limit oscillation in a good way. This system tends to be stable. It has almost no steady state error, good dynamic performance and steady state accuracy.

\section{Conclusion}

Aiming at the nonlinear system of the test rig, which can cause the anti-interference ability and the adaptive ability of the system are poor. Classic PID control cannot solve these problems well. In this paper, we design an adaptive control method based on RBF neural network. The simulation results of the RBF neural network PID control method show that, this method can improve the dynamic characteristics, reduce the steady-state error, shorten the rise time of the system, satisfies the requirement of control performance of the system.

\section{Acknowledgement}

The authors gratefully thank the Youth Foundation of the Henan University of Science and Technology (No. 2014QN024) and the Project of Basic and Advanced Technology Research of Henan Province of China (No. 162300410030) for financial support. This work is also supported by the Key Research Program of the Higher Education Institutions of Henan Province (No. 15A460022), and the Program for Innovative Research Team (in Science and Technology) in University of Henan Province (No. 15IRTSTHN008).

\section{References}

[1] Wang Zhanlin. Modern hydraulic control [M]. Beijing: Mechanical Industry Press, 1997.

[2] Chen Hemei. Research on hydraulic loading system of a certain type of aviation bearing test bench [D]. Central South University, 2010.

[3] Wang Ping. Design and research of axial servo loading test system [D]. Zhejiang University, 2012.

[4] Tang Kun, Wang Pingjun, Wu Wei et al. Nonlinear modeling and Simulation of servo system based on [J]. Simulink micro computer information, 2010.

[5] Yao Jianming. Research on intelligent control of electro hydraulic servo system based on neural network [D]. Taiyuan University of Technology, 2005.

[6] Newton DA. Design and Implementation of a Neural Network Controlled Electro-hydraulic 
Drave. Proc lnstn MechEngrs, 1994, 208:31-42.

[7] Ye Jun, Lou Guo. Neural network control of electro hydraulic servo system under nonlinear disturbance [J]. Hydraulic and pneumatic, 2005, (4).

[8] Yuan Zhaohui, Shi Changhai. Based on RBF neural network for electro-hydraulic servo system applied research [J]. Mechanical and electrical integration, 2008, 14(3):54-58. (8):65-69.

[9] Liu Jinkun. Intelligent control [M]. Beijing: Publishing House of electronics industry, 2005:171-176. 